\title{
BROA: An agent-based model to recommend relevant Learning Objects from Repository Federations adapted to learner profile
}

\author{
${ }^{1,2}$ Paula A. Rodríguez, ${ }^{1,2}$ Valentina Tabares, ${ }^{2}$ Néstor D. Duque, ${ }^{1}$ Demetrio A. Ovalle, ${ }^{3}$ Rosa M. Vicari \\ ${ }^{1}$ Departamento de Ciencias de la Computación y la Decisión, Universidad Nacional de Colombia- \\ Medellín, Colombia \\ ${ }^{2}$ Departamento de Informática y Computación, Universidad Nacional de Colombia-Manizales, \\ Colombia \\ ${ }^{3}$ Instituto de Informática, Universidade Federal do Rio Grande do Sul, Brasil
}

\begin{abstract}
Learning Objects (LOs) are distinguished from traditional educational resources for their easy and quickly availability through Web-based repositories, from which they are accessed through their metadata. In addition, having a user profile allows an educational recommender system to help the learner to find the most relevant LOs based on their needs and preferences. The aim of this paper is to propose an agent-based model so-called BROA to recommend relevant LOs recovered from Repository Federations as well as LOs adapted to learner profile. The model proposed uses both role and service models of GAIA methodology, and the analysis models of the MASCommonKADS methodology. A prototype was built based on this model and validated to obtain some assessing results that are finally presented.
\end{abstract}

Keywords - Artificial Intelligent in Education, GAIA, Learning objects repository federations, MAS-CommonKADS, Multi-agent Systems, Student-centered recommender systems

\section{INTRODUCTION}

$\mathrm{T}$ HE growth of digital information, high-speed computing, and ubiquitous networks has allowed for accessing to more information and thousands of educational re-sources. This fact has led to the design of new teachinglearning proposals, to share educational materials, and also to navigate through them [1]. Learning Objects (LOs) are distinguished from traditional educational resources for their easy and quickly availability through Web-based repository, from which they are accessed through their metadata. In order to maximize the number of LOs to which a student could have access, to support his/her teaching-learning process, digital repositories have been linked through centralized repository federations sharing in this way educational resources and accessing resources from others [2]. LOs must be tagged with metadata so that they can be located and used for educational purposes in Web-based environments [3]. Recommender systems are widely used online in order to assist users to find relevant information [4]. Having a user profile allows a recommender system to help the student to find the most relevant LOs based on the student's needs and preferences. Intelligent agents are entities that have sufficient autonomy and intelligence to be able to handle specific tasks with little or no human supervision [5]. These agents are currently being used almost as much as traditional systems, making it a good choice to solve problems where autonomous systems are required and thus they work not only individually but also cooperate with other systems to achieve a common goal. The aim of this paper is to propose a model for LO searching, retrieving, recommendation, and evaluator modeled through the paradigm of multi-agent systems from repository federations. For doing so, the searching process needs a query string that is entered by the user and a similar relevance user profile according to the student's learning style (LS). The LO searching process is performed using local and remote repositories, or repository federations, that are accessible via web along with LO descriptive metadata. Since LO Repositories (LORs) are distributed, are different in design and structure, and not handle the same metadata standards. There is also a coordinator to be responsible for directing the search to different repositories according to their characteristics. The recommendation is made through collaborative filtering, searching for a similar profile to the user who is doing the quest to deliver a user pair LOs evaluated positively.

The rest of the paper is organized as follows: Section 2 outlines main concepts involved in this research. Section 3 describes some related works to the model pro-posed. Section 4 introduces the multi-agent model proposal based on both role and service models of GAIA methodology, and the analysis models of the MAS-CommonKADS methodology. A validation of the system's operation can be visualized is shown in Section 5. Finally, conclusions and future work are presented in Section 6.

\section{BASIC CONCEPTS}

\section{A. Learning objects, repositories and federations}

According to the IEEE, a LO can be defined as a digital 
entity involving educational design characteristics. Each LO can be used, reused or referenced during computer-supported learning processes, aiming at generating knowledge and competences based on student's needs. LOs have functional requirements such as accessibility, reuse, and interoperability [6][7]. The concept of LO requires understanding of how people learn, since this issue directly affects the LO design in each of its three dimensions: pedagogical, didactic, and technological [7]. In addition, LOs have metadata that describe and identify the educational resources involved and facilitate their searching and retrieval. LORs, composed of thousands of LOs, can be defined as specialized digital libraries storing several types of resources heterogeneous, are currently being used in various e-learning environments and belong mainly to education-al institutions [8]. Federation of LORs serve to provide educational applications of uniform administration in order to search, retrieve, and access specific LO contents available in whatever of LOR groups [9].

\section{B. Recommender Systems}

Recommender systems are aimed to provide users with search results close to their needs, making predictions of their preferences and delivering those items that could be closer than expected [10],[11]. In the context of LOs these systems seeks to make recommendations according to the student's characteristics and its learning needs. In order to improve recommendations, recommender systems must perform feedback processes and implement mechanisms that enable them to obtain a large amount of information about users and how they use the LOs [2],[12].

\section{Multi-Agent Systems}

Agents are entities that have autonomy in order to perform tasks by achieving their objectives without human supervision. The desirable characteristics of the agents are as follows [13]: Reactivity: they respond promptly to perceived changes in their environment; Proactivity: agents can take initiative; Cooperation and Coordination: they perform tasks communicating with other agents through a common language; Autonomy: agents do not require direct intervention of humans to operate; Deliberation: they perform reasoning processes to make decisions, Distribution of Tasks: each agent has definite limits and identified the problems to be solved; Mobility: they can move from one machine to another over a network; Adaptation: depending on changes in their environment they can improve their performance, and Parallelism: agents can improve performance depending on changes in their environment.

Multi-agent Systems (MAS) are composed of a set of agents that operate and interact in an environment to solve a specific and complex problem. This paradigm provides a new way of analysis, design, and implementation of complex software systems and has been used for the development of recommender systems [14].

\section{Student Profile}

The student profile stores information about the learner, its characteristics and preferences, which can be used to obtain search results according to its specificity. To handle a user profile can be used to support a student or a teacher in the LO selection according to its personal characteristics and preferences [14]. Gonzalez et al. [15] include in the student profile contextual characteristics that can be seen as transient values that are associated with environmental changes during one student's learning system session along with different physical and technological variables. Duque (2009) presents a combination of VARK y FSLSM models with good results to characterize the students profile and thus, provide students with learning materials tailored to their specific learning styles [16].

\section{RELATED WORKS}

Morales et al. (2007) present an architecture based on the multi-agent paradigm to identify and retrieve relevant LOs using the information request supplied by the user. In addition, this proposal includes aspects related to quality of LOs which are specified within their semantic description to improve the LO selection [17]. Authors propose a multi-agent architecture to retrieve LOs, however they do not use student cognitive characteristics such as learning styles in order to make recommendations.

Gerling (2009) proposes an intelligent system to assist a user in finding appropriate LO, according to the search's subject by using the user profile which takes into account its characteristics, preferences, and LO relative importance. The recommender system incorporates an intelligent agent in order to retrieve educational resources on the Web, considering student's learning style [18]. However, the system's design only considers the utilization of one intelligent agent.

Duque (2009), in his doctoral thesis, proposes a multi-agent system for adaptive course generation. The system is composed of several intelligent agents: an agent for the student profile information; a domain agent, having the structure of the virtual course and the teaching material (TM); a HTN planner agent, and finally, a TM recovery agent which makes the process of TM search and retrieval [16]. This work focuses on creating customized virtual courses taking into account student's learning styles; however, it does not focus on LOs.

Prieta (2010) proposes a multi-agent architecture for the process of search and retrieval of LO in distributed repositories. An additional functionality is provided to the system is making the LORs statistics on the number of results found and response time, then make future consultations in the LORs rated [19]. This system offers neither recommendations to the user nor customized searches based on the LO metadata. Casali (2011) presents an architecture and implementation of a recommender system prototype based on intelligent agents, whose goal is to return an ordered list of the most appropriate LOs according to the parameters that characterizes the user profile, language preferences and the interaction degree that the user wants to have with the LO. The search is performed in repositories having descriptive LO metadata which involves 
educational characteristics [14]. The main limitation of this research is that although some student characteristics are considered into the user profile the user learning styles were not taken into account.

\section{MODEL PROPOSED}

BROA (Spanish acronym for Learning Object Search, Retrieval \& Recommender System) is a multi-agent system for searching, retrieving, recommendation and evaluator of LO, according to a search string entered by the user. The LOs resulting from the search are recommended based on the student's style of learning and other users' assessments. The Web-based LO search is performed over local and remote repositories, or by using LO repository federations through metadata descriptive LOs. Considering that LORs are distributed, they are different in design and structure, and hence they do not handle the same metadata standards. BROA was built under the MAS approach in order to exploit their advantages as follows: the Parallelism of Tasks for simultaneously searching in both local and remote LOR; the Deliberation ability for making decisions on which of LORs must perform the search and for performing user recommendations; Cooperation, Coordination and Distribution of tasks among agents by clearly identifying the problems to be solved by each agent and to define its limits. In our model each agent knows how LOs are stored and how each LO can be searched, accessed, and retrieved.

\section{A. Development Methodology}

There are different kinds of methodologies for modeling MAS, such as GAIA characterized for analyzing and designing agent-oriented systems. The main key concepts of GAIA are the following: roles, which are associated with responsibilities, permissions, activities, and protocols [5]. Another well-known MAS design methodology is MAS-CommonKADS proposed by Iglesias in his doctoral thesis [20] which integrates knowledge and software engineering along with objectoriented protocols. An integration of both methodologies was used in order to model the BROA system. The following is a brief description of each of these models: Role Model (GAIA): Allows the system designer to identify the expected functions of each of the entities that composes the system (goals, responsibilities, capabilities, and permissions). Service Model (GAIA): This model identifies all the services associated with each of the roles, its inputs, outputs, pre-conditions, and postconditions. Agent Model: Describes the characteristics of each agent, specifying name, kind of agent, role, description, skills, services, activities, and goals. According to the GAIA methodology an agent can play several roles as shown in Figure 1, thus, a changing role diagram must be used in this case. Task Model: This model describes all the tasks that agents can perform along with the objectives of each task, its decomposition, and troubleshooting methods to solve each objective. Figure 2 shows the BROA's task diagram. Expertise Model: Describes the ontologies (knowledge and its relationships) that agents need to achieve their objectives.
Communication Model: Describes main interactions among humans and software agents along with human factors involved for the development of these interfaces. Organization Model: This model aims to describe the human organization in which the multi-agent system is involved along with the software agent organization structure. Coordination Model: Dynamic relationships among software agents are expressesed through this model. For doing so, all the conversations among agents must be described: interactions, protocols, and capabilities required. Figure 3 shows the BROA's sequence diagram that specifies main interactions among agents.

\section{B. BROA's Architecture}

The design phase of MAS-CommonKADS methodology takes as input all the models got from the analysis phase and transforms their specifications for implementation. In addition, the architecture of each agent and the global network architecture must be provided [20].

Figure 4 shows the multi-agent architecture of the model proposed. This architecture was used to develop the BROA

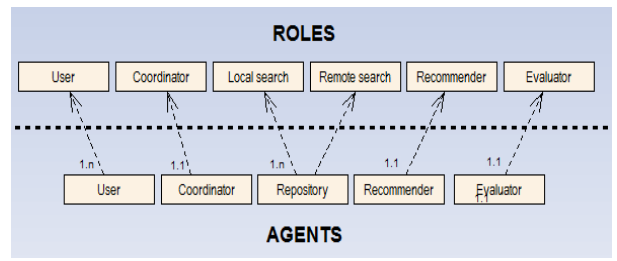

Fig. 1. Transformation of roles in agent's diagram

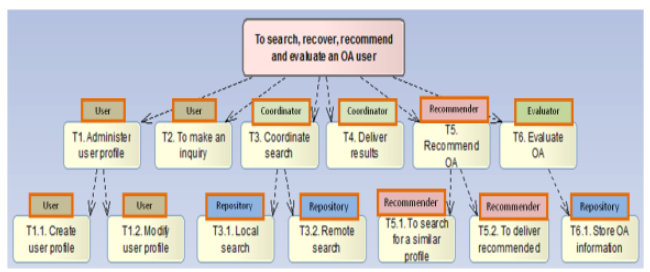

Fig. 2. Task Diagram

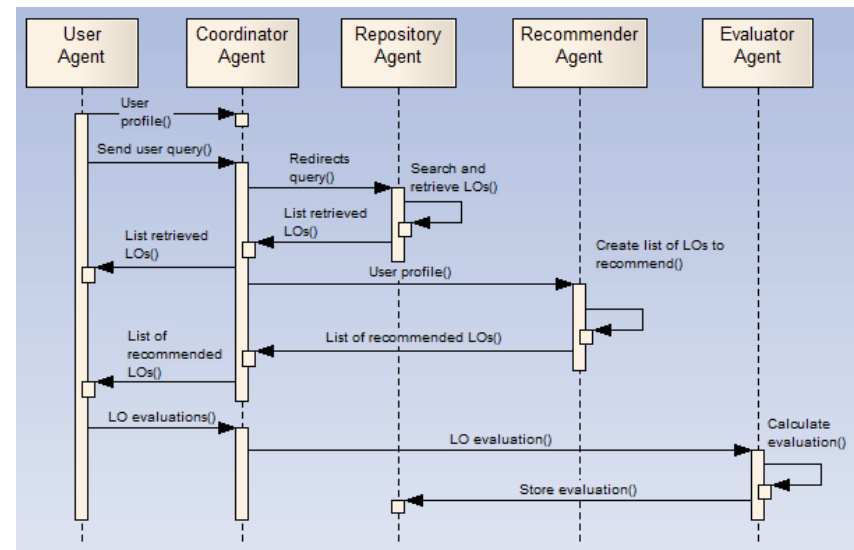

Fig. 3. Sequence diagram

system, implemented using JADE (Java Agent Development Framework) agents [21]. The next Section describes each of the agents of the BROA system along with the main interactions that exist among them. 


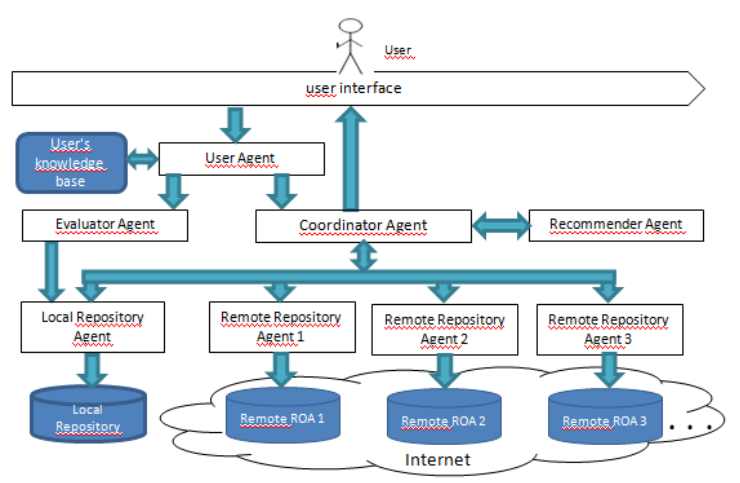

Fig. 4. BROA/SRLO's Architecture

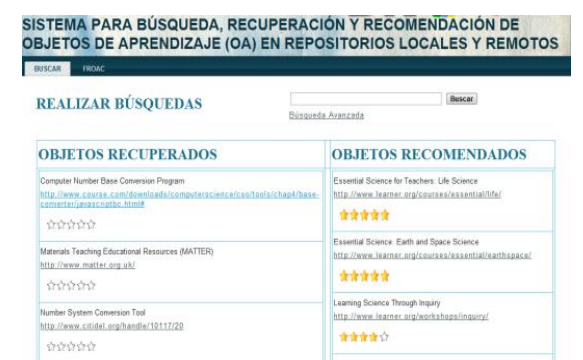

Fig. 5. BROA/SRLO's Web-based Interface

\section{Agent Description}

User Agent: This agent communicates directly with the human user and whose role is representing him within the system along with communications with other agents (Coordinator and Evaluator). Also, the user agent manages the user's profile, enabling the creation and modification of profile's characteristics and preferences. Finally, this agent sends the query string to the coordinator agent in order to perform the LO search and the evaluation of the recommended LOs. Coordinator Agent: This agent is of deliberative nature since it takes care of redirectioning queries that the user makes to both the local and the remote repositories. This agent knows the repositories associated with the system and the information that each of them manages. In addition, it can access the user agent's profile to know what characteristics are useful for making a recommendation (learning style, educational level, language preference, among others). Local and Remote Repository Agent: Repository agents are responsible for making accurate LO searches in both local and remote repositories. This agent recognizes how LOs are stored inside the repositories, under what standard and type of metadata that manages. Also knows the type of search that can be performed within the repository and how to recover a particular LO stored. The local repository agent is also responsible for storing the LO evaluation given from an evaluator agent. Similarly, in the proposed architecture there is a repository for each LOR agent federation is local or remote. Recommender Agent: This agent makes two recommendations; the first stage is to find users registered in the system with similar profile to the user so having the same learning style and education level. The LOs selected by those users having a score greater or equal than 4 are shown. The second stage of recommendation is based on LOs recovered in all different repositories, based on user's LO query. This recommendation is based on the student's learning style. It is important to highlight that in the model proposed, the recommendation is based on the metadata that describes the LO and the information of learning style, educational level, and language preference of the registered student. In order to represent the agent's knowledge production rules were used, such as the following rule:

\section{LearningStyle (Visual-Global)^ \\ LearningResourceType (figure) $\vee$ \\ LearningResourceType(graph) $\vee$ \\ LearningResourceType(slide) $\vee$ \\ LearningResourceType(table)^ \\ InteractivityLevel(medium) $\vee$ \\ InteractivityLevel(high).}

When there is a failure of similar users, the system shows only the results of the second recommendation and then stored the user profile information and evaluated LOs, within the knowledge base. Evaluator agent: This agent manages the evaluation performed by a user to some of the LOs that have been explored. The evaluation is made through explicit qualification that is given by the selected student who rates the specific LO from 1 to 5 according to his/her own satisfaction level.

\section{Platform Design}

The BROA's agent architecture was developed in JAVA, using JDOM for handling XML user's profiles. The local repository manager is stored in the PostgreSQL database that is characterized to be stable, with high performance, and great flexibility. The agent creation and management is made by using JADE platform using FIPA-ACL performatives [21]. The ontology creation was performed by using Protégé and finally, the Web integration was made based on the ZK framework. Figure 5 shows BROA's Web interface with the recommended and retrieved LOs. For the LO search process there was a student who had a Visual-sequential learning style and the search string used was: "computer science". Thus, a total of 196 LOs were recovered and only recommended, after a "learning style" filtering just 45 of them.

\section{V.EXPERIMENTS AND RESULTS}

BROA system provides to the human user LO lists by using its interface. The first list is the result of the search made by the user according to his criteria. The second list presents list of recommended items to the user, which correspond to those LOs that are the most adapted to his own learning style.

To validate the BROA system a test was performed based on the keyword "computer science". In addition, a comparison was made with the results given by the system concerning the LOs recommended for users with different learning styles proposed by Duque [16]: Visual-Global, Visual-sequential, Auditory-Global, Auditory-sequential, Kinesthetic-Global, Kinesthetic-sequential, Reader-Global, Reader-sequential. 
Thus, virtual users with different profiles were generated and LOs from real repositories were recovered. Figure 6 shows the results got for the tests being performed. A total of 196 LOs were retrieved after the search process for students with different learning styles. Figure 5 shows the quantity of LOs for each of the different learning styles. The BROA system makes a good recommendation process since the LOs provided are well adapted to the student's learning profile.

In order to evaluate the results of recommendations given by the system the Precision measure [22] was used which purpose is to analyze the quality of the retrieval.

$$
\text { Precision }=\frac{\text { Relevant LOs }}{\text { RelevantLOs+Retrieved LOs }}
$$

Figure 6 shows the results obtained by applying the Precision measure formula (1) to each learning style and additionally comparing values obtained with and without

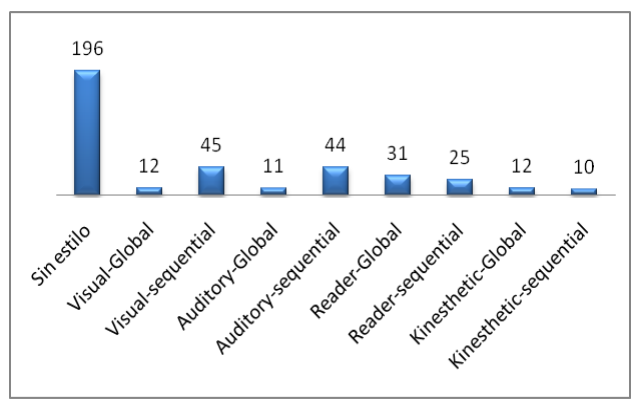

Fig. 5. LOs retrieved after search

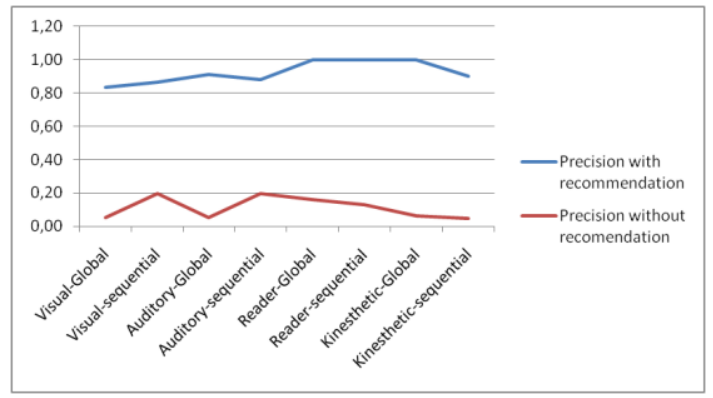

Fig. 6. Precision

recommendation when accessing LO Merlot repository.

\section{CONCLUSION AND FUTURE WORK}

This paper proposes a model for learning object searching, retrieving, recommendation, and evaluation modeled through the paradigm of MAS from repository federations. The BROA (Spanish acronym for Learning Object Search, Retrieval \& Recommender System) system was built using this model. There is an agent in the BROA system dedicated to each repository accessed by the system. In order to facilitate LO searches, the agent knows how the LOs are stored, how is the way of accessing and recovering them, under what standard and type of metadata the LOs are stored and handled by the specific repository. Those searches are performed in a local LOR, where the already evaluated LOs are stored, and also performed in remote LORs associated to the system. The BROA system offers two types of recommendation; the first one is based on finding similar profiles. This first recommendation approach has not already been implemented in this prototype. The second type of recommendation is by searching the metadata of the LO, taking into account the query performed by the user, the results are presented at the right side on Figure 5. The model proposed in this paper addressed issues such as working on LOs and learning styles and making recommendations by the system to the user based on customized searches using the LO metadata. In addition, the problem modeling using a MAS technique was an excellent option, which allowed the disintegration into functional blocks, without losing the systemic point of view, which leads to distributing the solution in diverse entities that require specific knowledge, processing and communication between each other. The MAS allowed a neutral vision in the model proposed.

It is envisaged as future work to add an interface agent to make context-aware adaptations, along with the list of LOs delivered by the system considering other issues such as type of device from where the query is made, bandwidth, among others. For the evaluation process, it is intended to make templates for the user to rate its opinion about recommended LOs (explicit evaluation). The agent should analyze the results of the explicit evaluation and use logs, to assign a rating to each LO. Also it is envisaged to improve the theoretical and practical basis of the first stage of recommendations made by the system through collaborative filtering techniques. The learning style for this prototype should be selected by the user, an additional future work aims to propose a learning style test that will define which kind of learning style the user who is logged in the system has.

\section{ACKNOWLEDGMENT}

The research reported in this paper was funded in part by the COLCIENCIAS project entitled "ROAC Creación de un modelo para la Federación de OA en Colombia que permita su integración a confederaciones internacionales" Universidad Nacional de Colombia, with code 1119-521-29361.

\section{REFERENCES}

[1] C. I. Peña, J.-L. Marzo, J. L. De la Rosa, and R. Fabregat, "Un Sistema de Tutoría Inteligente Adaptativo Considerando Estilos de Aprendizaje," VI Congreso Iberoamericano de Informática Educativa, pp. $1-12,2002$.

[2] J. Z. Li, "Quality, Evaluation and Recommendation for Learning Object," International Conference on Educational and Information Technology (ICEIT 2010), pp. 533-537, 2010.

[3] A. B. Gil and F. García, "Un Sistema Multiagente de Recuperación de Objetos de Aprendizaje con Atributos de Contexto," ZOCO'07/CAEPIA, 2007.

[4] K. Niemann, M. Scheffel, M. Friedrich, U. Kirschenmann, H.-C. Schmitz, and M. Wolpers, "Usage-based Object Similarity," Journal of Universal Computer Science, vol. 16, no. 16, pp. 2272-2290, 2010.

[5] M. Wooldridge, N. R. Jennings, and D. Kinny, "The Gaia Methodology for Agent-Oriented Analysis and Design," Autonomous Agents and Multi-Agent Systems, vol. 3, pp. 285 - 312, 2000. 
[6] Y. Ouyang and M. Zhu, "eLORM: Learning Object Relationship Mining-based Repository," Online Information Review, vol. 32, no. 2, pp. 254-265, 2008.

[7] D. Betancur, J. Moreno, and D. Ovalle, "Modelo para la Recomendación y Recuperación de Objetos de Aprendizaje en Entornos Virtuales de Enseñanza/Aprendizaje," Avances en Sistemas e Informática, vol. 6, no. 1, pp. 45-56, 2009.

[8] F. De Prieta and A. B. Gil, "A Multi-agent System that Searches for Learning Objects in Heterogeneous Repositories," Advances in Intelligent and Soft Computing, vol. 71, pp. 355-362, 2010.

[9] H. Van de Sompel, R. Chute, and P. Hochstenbach, "The aDORe Federation Architecture: Digital Repositories at Scale," International Journal on Digital Libraries, vol. 9, no. 2, pp. 83-100, 2008.

[10] F. Chesani, "Recommendation Systems," Corso di laurea in Ingegneria Informatica, pp. 1-32, 2007.

[11] K. Mizhquero and J. Barrera, “Análisis, Diseño e Implementación de un Sistema Adaptivo de Recomendación de Información Basado en Mashups," Revista Tecnológica ESPOL-RTE, 2009.

[12] O. Sanjuán, E. Torres, H. Castán, R. Gonzalez, C. Pelayo, and L. Rodriguez, "Viabilidad de la Aplicación de Sistemas de Recomendación a entornos de e-learning," 2009.

[13] N. R. Jennings, "On Agent-based Software Engineering," Artificial Intelligence, vol. 117, no. 2, pp. 277-296, Mar. 2000.

[14] A. Casali, V. Gerling, C. Deco, and C. Bender, "Sistema Inteligente para la Recomendación de Objetos de Aprendizaje," Revista Generación Digital, vol. 9, no. 1, pp. 88-95, 2011.

[15] H. González, N. Duque Méndez, and D. Ovalle C., "Técnicas Inteligentes para la Actualización Dinámica del Perfil del Usuario en un Sistema de Educación Virtual," in Tendencias en Ingeniería de Software e Inteligencia Artificial, 2009.

[16] N. Duque, "Modelo Adaptativo Multi-Agente para la Planificación y Ejecución de Cursos Virtuales Personalizados - Tesis Doctoral," Universidad Nacional de Colombia, 2009.

[17] E. Morales and A. Gil, "Arquitectura para la Recuperación de Objetos de Aprendizaje de Calidad en Repositorios Distribuidos," SCHA: Sistemas Hipermedia Colaborativos y Adaptativos. II Congreso Español de Informática CEDI 2007, vol. 1, no. 1, pp. 31 - 38, 2007.

[18] V. B. Gerling, "Un Sistema Inteligente para Asistir la Búsqueda Personalizada de Objetos de Aprendizaje," Universidad Nacional de Rosario, 2009.

[19] F. de la Prieta, A. G. González, J. M. Corchado, and E. Sanz, "Sistema Multiagente Orientado a la Búsqueda, Recuperación y Filtrado de Objetos Digitales Educativos," VIII Jornadas de Aplicaciones y Transferencia Tecnológica de la Inteligencia Artificial, pp. 65 - 74, 2010.

[20] C. Á. Iglesias Fernández, "Definición de una Metodología para el Desarrollo de Sistemas Multiagentes," Universidad Politécnica de Madrid, 1998.

[21] F. Bellifemine, A. Poggi, and G. Rimassa, "JADE - A FIPA-compliant Agent Framework," Proceedings of PAAM, 1999.

[22] G. Shani and A. Gunawardana, "Evaluating Recommendation Systems," Recommender Systems Handbook, pp. 257 - 297, 2011.

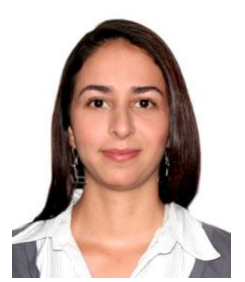

Paula A. Rodriguez is Manager of Information Systems from Universidad Nacional de Colombia, Manizales and currently she advances Masters Studies in Systems Engineering program from Universidad Nacional de Colombia. She participates in the Research Group in Adaptive Intelligent Environments GAIA. Her research interests are in the areas of Artificial Intelligence in Education and Multi-Agent Systems (MAS), Learning Object Repository Federations, among others.

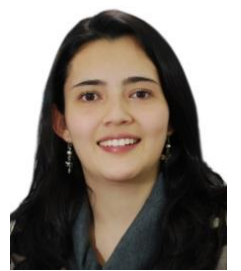

Valentina Tabares is Manager of Information Systems from Universidad Nacional de Colombia, Manizales and currently she advances Masters Studies in Systems Engineering program from Universidad Nacional de Colombia. She participates in the Research Group in Adaptive Intelligent Environments GAIA. Her research interests are in the areas of Artificial Intelligence in Education and Multi-Agent Systems (MAS), Learning Object Repository Federations, among others.

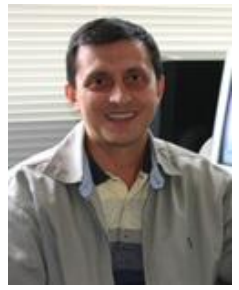

Néstor D. Duque is Associate Professor from Universidad Nacional de Colombia, campus Manizales and head of the Research Group in Adaptive Intelligent Environments GAIA. He received his master's degree in Systems Engineering from Universidad Nacional de Colombia, and his $\mathrm{PhD}$ in Engineering from Universidad Nacional de Colombia, campus Medellin. His $\mathrm{PhD}$ thesis was entitled "Adaptive Multi-Agent Model for Planning and Execution of Adaptive Virtual Courses" with Cum Laude honors. Author of a number of articles in scientific journals and book chapters including topics on their research and academic work, speaker at major national and international events; head in the development process of national and international research projects, member of academic committees of a dozen national and international journals, academic review in post-graduate academic programs and special events. He has also received some meritorious distinction for researching and teaching from School of Administration at Universidad Nacional de Colombia, campus Manizales.

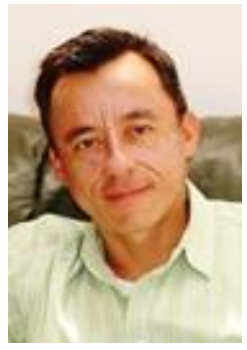

Demetrio A. Ovalle is Full Professor at Universidad Nacional de Colombia, campus Medellin. He was head of the Department of Computer Science at School of Mines (2006-2008). He is presently head of GIDIA (Artificial Intelligence Research \& Development Group), categorized as A1, maximum category established by Colombian National Science \& Technology Bureau. He has published over 150 refereed publications in Journals, Books and International Conferences. He received his Master's and $\mathrm{PhD}$ degrees in Informatics respectively from Institut National Polythechnique in 1987, and Université Jospeh Fourier in 1991, in Grenoble, France. His research interests are in the areas of Distributed Artificial Intelligence and Multi-Agent Systems (MAS), Virtual Learning Environments, Intelligent Tutoring Systems, Mobile \& Ubiquitous Computing, Ambient Intelligence, Autonomic Systems and Wireless Sensor Network. He has also received some meritorious distinctions in research from School of Mines at Universidad Nacional de Colombia, campus Medellin.

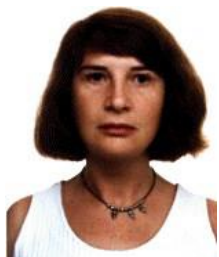

Rosa M Vicari is Full Professor at Federal University of Rio Grande do Sul (UFRGS), Brazil. She received her PhD in Computer Science at University of Coimbra, Portugal. Her research interests are in the areas of Artificial Intelligence in Education and Multi-Agent Systems (MAS), Virtual Learning Environments, Intelligent Tutoring Systems, Semantic Web and Ontologies, Learning Object Repository Federations, among others. She has also received some meritorious distinctions in research from UFRGS 